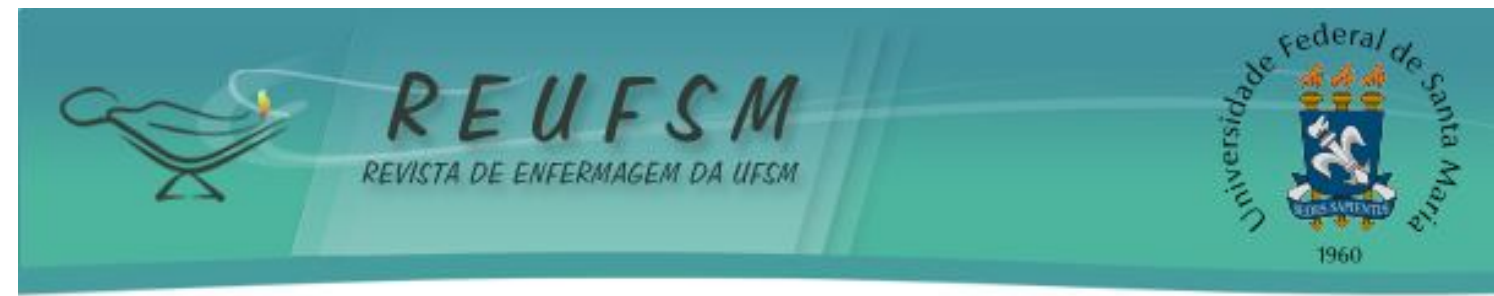

ARTIGO DE REVISÃO

\title{
CRIANÇAS COM NECESSIDADES ESPECIAIS DE SAÚDE: TENDÊNCIAS DAS PESQUISAS EM ENFERMAGEM
}

\section{CHILDREN WITH SPECIAL HEALTH CARE NEEDS: RESEARCH TRENDS IN NURSING NIÑOS CON NECESIDADES ESPECIALES DE SALUD: TENDENCIAS DE LA INVESTIGACIÓN EN ENFERMERÍA}

Andressa da Silveira ${ }^{1}$ Eliane Tatsch Neves ${ }^{2}$

\begin{abstract}
RESUMO: Pesquisa bibliográfica desenvolvida no Banco de Teses e Dissertações da Associação Brasileira de Enfermagem entre 2001 e 2008 utilizando as palavras enfermagem pediátrica e família. Objetivou-se identificar e analisar as pesquisas de enfermagem que abordam a temática do cuidado de crianças com doença crônica e/ ou incapacitante no domicílio. Foram encontrados 311 resumos e após aplicar os critérios de inclusão e exclusão foram selecionadas 36 produções que foram submetidas à análise descritiva e a análise de conteúdo temática. Constatou-se que a tendência dos estudos está voltada para a família da criança doente, a doença e a hospitalização. Porém, identificaram-se lacunas no que tange ao preparo destas famílias para dar continuidade ao tratamento e aos cuidados no domicílio. Recomenda-se comprometimento e capacitação constante dos profissionais para assistir as crianças dependentes de tecnologia e seus familiares.
\end{abstract}

Descritores: Enfermagem pediátrica; Família; Educação em saúde; Doença crônica.

ABSTRACT: Bibliographical research developed at the Bank of Thesis of Associação Brasileira de Enfermagem from 2001 to 2008 using the words: pediatric nursing and family. The objective was to identify and analyze nursing research about children care that involves chronic disease and/ or disability at home. 311 abstracts were found and after applying the inclusion and exclusion criteria, 36 productions were selected. Data were submitted to descriptive analysis and thematic content analysis. It was found that the trend of the studies is focused on the family, on the sick child, illness and hospitalization. However, we identified gaps in relation to the preparation of these families to continue the treatment and care at home. Commitment and constant training of professionals to assist technology-dependent children and their families are recommended.

Descriptors: Pediatric nursing; Family; Health education; Chronic disease.

RESUMEN: Investigación bibliográfica desarrollada en el Banco de Tesis de la Associação Brasileira de Enfermagem, entre 2001 y 2008, en la que se utilizaron las palabras enfermería pediátrica y familia. El objetivo fue identificar y analizar como la investigación en enfermería ha tratado de la cuestión de la atención a los niños con enfermedades crónicas y/o discapacitantes en la residencia. Se encontraron 311 resúmenes y, después de sometidos a criterios de inclusión y exclusión, se seleccionaron 36 producciones, de las que se hizo un análisis descriptivo y del contenido temático. Se encontró que la tendencia de los estudios se centra en la familia del niño enfermo, la

\footnotetext{
${ }^{1}$ Enfermeira. Mestranda do Programa de Pós Graduação em Enfermagem da Universidade Federal de Santa Maria (UFSM), Bolsista REUNI. E-mail: andressadasilveira@gmail.com

2 Enfermeira Pediatra. Doutora em Enfermagem, Professora Adjunta do Curso de Enfermagem e do Programa de Pós Graduação em Enfermagem da Universidade Federal de Santa Maria (UFSM). E-mail: elianeves03@gmail.com
} 


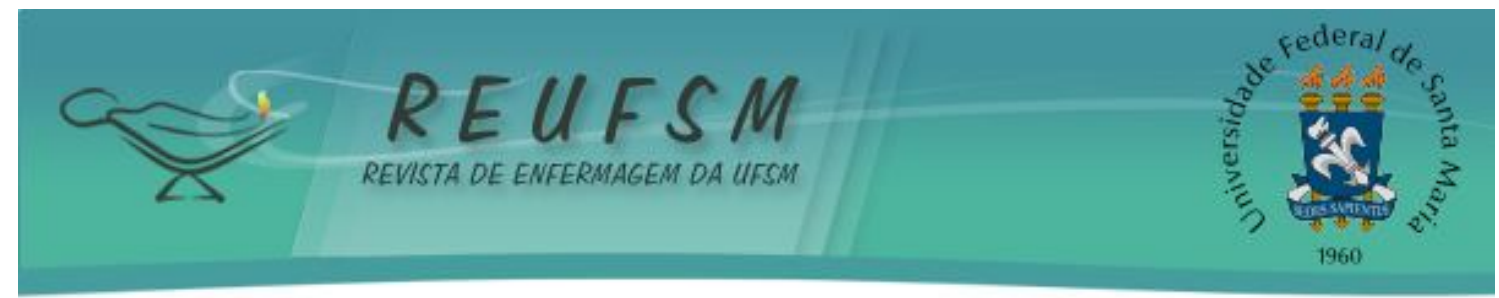

enfermedad y la hospitalización. Sin embargo, hemos identificado las Iagunas con relación a la preparación de esas familias para continuar con el tratamiento y la atención en el hogar. Se recomienda la formación y el compromiso constante de los profesionales para ayudar a los niños que dependen de la tecnología y a sus familias.

Descriptores: Enfermería pediátrica; Familia; Educación para la salud; Enfermedades crónicas.

\section{INTRODUÇÃO}

A saúde da criança passou por avanços científicos e políticos ao longo do tempo. No século XVII, a cada quatro crianças nascidas pelo menos uma morria antes de completar o primeiro ano de vida. ${ }^{1}$ A criança não tinha assistência específica, e somente a partir da fundação do primeiro hospital pediátrico em 1802 em Paris, foi implementada a qualidade da assistência em pediatria. ${ }^{2}$

No Brasil em 1984 foi criado o Programa de Assistência Integral à Saúde da Criança (PAISC). ${ }^{3}$ Já na década de 1990 a criança e o adolescente passam a ter seus direitos regulamentos a partir da criação do Estatuto da Criança e do Adol escente (ECA) por meio da Lei $8.069 / 90 .^{4}$

Destaca-se que as práticas de cuidado à criança se modificaram com o passar dos anos, sendo que os avanços tecnológicos na década de 1990 contribuíram para a el evação da sobrevida de crianças com doenças de alto nível de complexidade. Neste contexto, a terapia intensiva neonatal e pediátrica, ao mesmo tempo em que possibilita expectativa de vida para essas crianças, acaba por gerar um novo grupo de crianças clinicamente frágeis e, por vezes, dependente de tecnologias. Esse grupo de crianças foi denominado, no Brasil, como crianças com necessidades especiais de saúde (CRIANES). ${ }^{5}$ Nos Estados Unidos, este grupo é estudado desde a década de 1980 e foi denominada pelo Maternal Children Bureau como Children with Special Health Care Needs (CSHCN) para designar as crianças com estado de saúde delicado além da dependência de cuidados de saúde contínuos para sobreviver. ${ }^{6}$

As CRIANES demandam de cuidados especiais de saúde, sejam eles de natureza temporária ou permanente, porém com uma pluralidade de diagnósticos e dependência dos serviços de saúde. Desta forma, cuidar de CRIANES requer conhecimento e preparo dos profissionais de saúde para que a criança tenha tratamento e cuidados adequados.

Salienta-se que os familiares das CRIANES devem dar continuidade ao tratamento no âmbito domiciliar, neste contexto, os mesmos precisam estar preparados. Assim, destaca-se a necessidade do familiar/ cuidador estar instrumentalizado para a prática de cuidado da CRIANES no domicílio.

Nesse sentido, a equipe de enfermagem precisa negociar os saberes e práticas de modo que a família possa contribuir nas múltiplas demandas de cuidados dessas crianças no domicílio. Consequentemente, mediar saberes necessários ao desenvolvimento do cuidado as CRIANES é uma tarefa complexa e necessária para a enfermagem que se depara com a invisibilidade dessa clientela nas taxas oficiais e nas políticas públicas específicas para essa demanda.

Sabe-se que o enfermeiro, em sua prática profissional competente, deve ser capaz de desenvolver ações educativas adequadas às reais necessidades dos indivíduos e dos grupos sociais, que permitam a transformação consciente da realidade. Assim, no cuidado à criança sempre é possível encontrar espaços para a educação em saúde, considerando que a mesma, deve permear todas as práticas do cuidado infantil e envolver seus familiares nesse processo. ${ }^{8-9}$ 


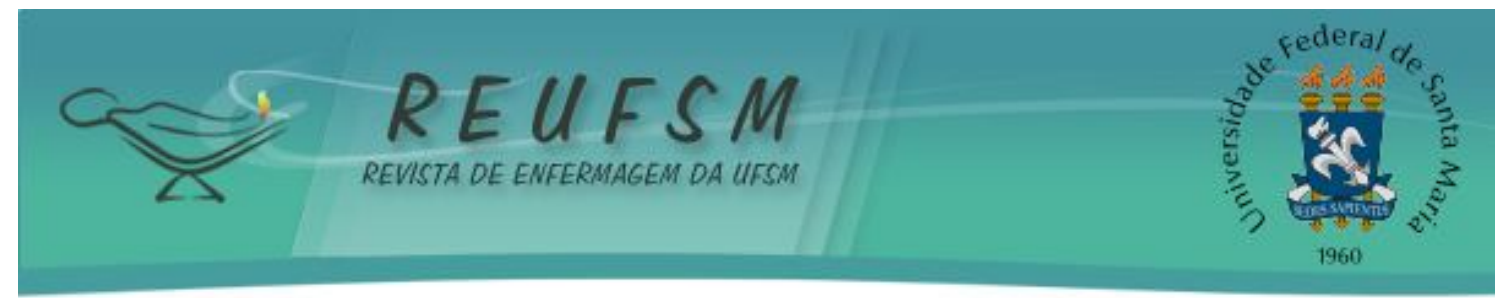

Diante do exposto, evidencia-se a necessidade de conhecer o que tem sido pesquisado por enfermeiros sobre a assistência as CRIANES, buscando compreender de que forma tem acontecido o cuidado destas crianças no domicílio.

Este estudo tem por objetivo identificar e analisar as pesquisas de enfermagem que abordam a temática do cuidado de crianças com doença crônica e/ ou incapacitante no domicílio.

\section{MATERIAIS E MÉTODO}

Trata-se de uma pesquisa bibliográfica desenvolvida no Banco de Dissertações e Teses da Associação Brasileira de Enfermagem (ABEN) no período compreendido entre os meses de outubro a novembro de 2010. Para busca dos resumos nos catálogos com 0 recorte temporal de 2001 a 2008 utilizaram-se as palavras enfermagem pediátrica e posteriormente família. Tendo em vista que crianças com doença incapacitante e/ ou dependente de tecnologia não são descritores, ampliou-se a busca para depois refiná-la com os critérios de inclusão e exclusão.

Foram excluídas as produções que após leitura do título e do resumo não convergiam com a temática proposta. Foram incluídas teses e dissertações que abordaram questões relacionadas à saúde da criança com doença incapacitante e/ ou dependente de tecnologia, CRIANES e cuidado prestado por familiares no domicílio.

Com as palavras enfermagem pediátrica e família foram encontradas um total de 311 resumos entre teses e dissertações, sendo 102 produções relativas à enfermagem pediátrica. Após aplicar os critérios de inclusão e exclusão foram selecionados 15 resumos. Com a palavra família foram encontradas 209 produções, foram aplicados critérios de inclusão e exclusão, sendo selecionados 21 resumos. Perfazendo um total de 36 produções que constituíram o corpus da pesquisa, e foram organizadas em um quadro sinóptico, contendo as seguintes variáveis: título, autor, suj eitos, cenário, objetivo, abordagem, tipo de publicação, instituição, estado e ano de publicação.

A análise teve um primeiro momento descritivo em que os dados foram tabulados e analisados sob forma de estatística simples, sendo apresentados em tabelas com a distribuição de frequências absolutas e relativas, e a seguir foi aplicada a análise de conteúdo. ${ }^{10}$

\section{RESULTADOS E DISCUSSÃO}

Quanto aos sujeitos que fizeram parte das pesquisas, salienta-se que $44,4 \%$ correspondem a famílias, $21,9 \%$ a enfermeiras/ equipe de enfermagem, $11,1 \%$ família/ equipe, 8,3\%a mães, 8,3\% a cuidadoras, 5,5\%a família/ criança.

Os resultados demonstram que a enfermagem percebe a necessidade e tem atuado na perspectiva da família, tornando-a um possível cliente para a assistência. Autores pontuam a necessidade de estudos que compreendam como o enfermeiro direciona-se para as famílias, importando-se em cuidar delas. ${ }^{11}$

Em relação à abordagem utilizada, $80,5 \%$ correspondem a pesquisas do tipo qualitativa, $13 \%$ não foi descrito a abordagem utilizada nos resumos, $2,7 \%$ são do tipo quantitativa, e outros $2,7 \%$ a pesquisa quali-quantitativa.

Sabe-se que a área da saúde tem mostrado interesse crescente por pesquisas qualitativas que na última década tornaram-se bem aceitas, no entanto, em épocas passadas pesquisas qualitativas eram consideradas não-científicas. ${ }^{12}$

A pesquisa qualitativa oferece maior liberdade para o pesquisador interpretar, porém, não poderá dar margem de dúvida sobre o que encontrou, e sobre o curso da interpretação. Deve- 


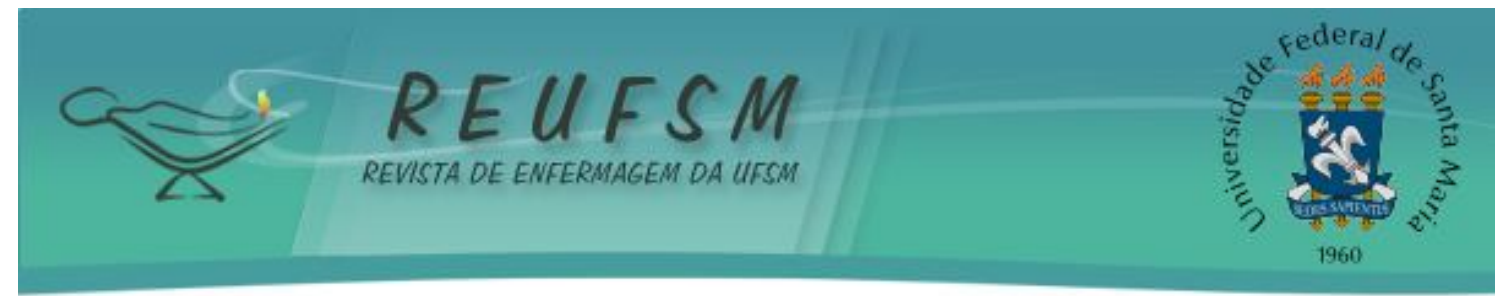

se considerar, ainda, que as pesquisas quantitativas passam por crivos rigorosos desde a formulação do problema até a comprovação da hipótese testada como resposta. ${ }^{13}$

Entretanto, alguns autores trazem a necessidade das duas abordagens para o meio científico, ressaltando tanto métodos qualitativos como quantitativos para conduzir a resultados sobre realidades sociais, e que não há sentido em atribuir maior relevância de um sobre 0 outro. ${ }^{10}$ Assim, podemos considerar que a abordagem qualitativa vem conquistando espaços nas pesquisas. Ressalta-se, ainda, a importância do pesquisador seguir o método adequado de acordo com o objeto de estudo proposto.

$\mathrm{Na}$ categoria correspondente ao cenário das pesquisas, salienta-se o cenário hospitalar com $69,4 \%$ em $11,1 \%$ não consta o cenário da pesquisa nos resumos, o domicílio aparece em 5,5\% das pesquisas, 5,5\% correspondem à comunidade, outros 5,5\% têm como cenário abrigo, sendo que 2,7\% ocorreram em casa de reabilitação.

A partir dos resultados pode-se constatar que embora maior parte dos estudos tenha sido desenvolvida no âmbito hospitalar, a enfermagem vem ampliando espaços de pesquisa no âmbito domiciliar, comunidade e abrigos.

Pesquisa realizada em 2009 ressalta que os campos de atuação na enfermagem têm crescido nos últimos anos, possibilitando novas perspectivas de conhecimento em múltiplas direções e espaços. ${ }^{14}$

Quanto ao objetivo do estudo $27,7 \%$ focaram no significado da doença e na hospitalização para a família; $16,6 \%$ centrado na família como cliente de enfermagem; $16,6 \%$ na família e equipe na prática de cuidado; e outros 16,6\% na educação em saúde. Destaca-se, ainda, $13,8 \%$ de produções que tem como objetivo a assistência à criança com necessidade especial de saúde. $\mathrm{E}$ em $8,3 \%$ o obj etivo do estudo não constava no resumo.

Em relação aos tipos de pesquisa publicadas, 69,4\% são dissertações de mestrado, e 30,6\% correspondem à tese de doutorado. Os dados demonstram um crescimento de pesquisadores na enfermagem, destacando-se a incidência de mestres em enfermagem em sua maioria.

Os autores trazem ainda, que a enfermagem enquanto profissão tem consolidado, por meio de pesquisas, a formação de um corpo teórico próprio que a visibilize e projete-a como ciência. ${ }^{14}$

No que diz respeito ao ano de publicação, considera-se o recorte temporal de oito anos compreendidos entre os anos de 2000 a 2008. Sendo que as pesquisas foram categorizadas de dois em dois anos, conforme tabela 1 a seguir:

Tabela 1 - Recorte temporal das pesquisas

\begin{tabular}{l|cc}
\hline Recorte temporal & N & $\%$ \\
\hline $2000-2002$ & 15 & 41,7 \\
$2003-2005$ & 11 & 30,6 \\
$2006-2008$ & 10 & 27,7 \\
\hline Total & 36 & 100 \\
\hline
\end{tabular}

Conforme os dados da tabela acima, pode-se dizer que o período entre os anos de 2000 a 2002 tem maior índice de pesquisas referentes à tese de doutorado e dissertação de mestrado. Possivelmente, essa el evação deva-se a observação, por parte dos enfermeiros em sua prática, do aumento do número de crianças com maior complexidade diagnóstica, incluindo-se aí a primeira geração de crianças portadoras de HIV/ Aids.

Em relação aos Estados de origem das pesquisas salienta-se São Paulo com 38, 8\%, Rio de J aneiro com 36,1\% Santa Catarina 8,3\% Paraíba 8,3\% Rio Grande do Sul 5,5\% e 2,7\% que tem como Estado de origem o Ceará. 


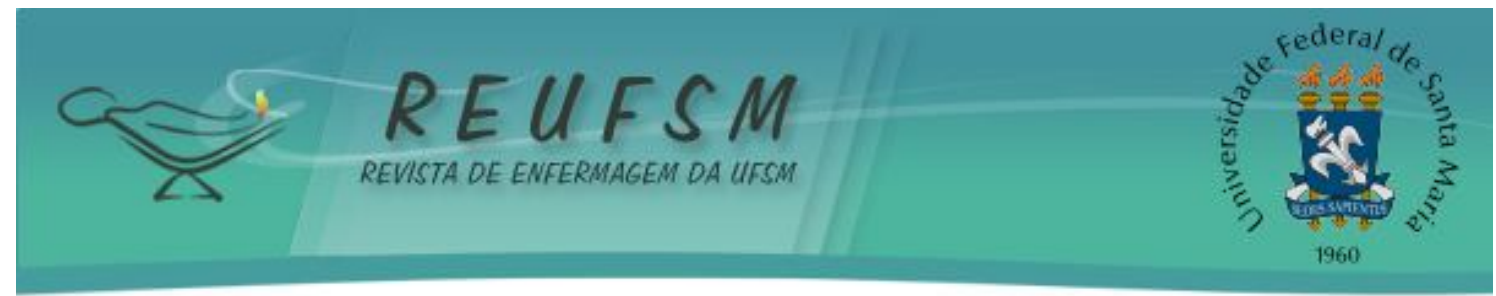

$\mathrm{Na}$ categoria Instituição, ressalta-se que apareceram 11 instituições com pesquisas de mestrado e doutorado, distribuídas em 6 estados brasileiros, conforme tabela 2 a seguir:

Tabela 2 - Instituições das pesquisas

\begin{tabular}{|c|c|c|}
\hline Instituição & $n$ & $\%$ \\
\hline Escola de Enfermagem Anna Nery - RJ & 9 & 25 \\
\hline Escola Paulista de Medicina - SP & 5 & 13,9 \\
\hline $\begin{array}{l}\text { Universidade de São Paulo campus Ribeirão Preto - } \\
\text { SP }\end{array}$ & 4 & 11,1 \\
\hline Escola de Enfermagem Alfredo Pinto - RJ & 4 & 11,1 \\
\hline $\begin{array}{l}\text { Escola de Enfermagem da Universidade de São } \\
\text { Paulo-SP }\end{array}$ & 4 & 11,1 \\
\hline Universidade Federal de Santa Catarina - SC & 3 & 8,3 \\
\hline Universidade Federal da Paraíba - PB & 3 & 8,3 \\
\hline Universidade Federal de Pelotas - RS & 1 & 2,8 \\
\hline Universidade de Passo Fundo - RS & 1 & 2,8 \\
\hline Universidade Federal do Ceará - CE & 1 & 2,8 \\
\hline Universidade Estadual de Campinas - SP & 1 & 2,8 \\
\hline Total & 36 & 100 \\
\hline
\end{tabular}

Por meio dos dados descritos na tabela 2, pode-se identificar que a região sudeste do Brasil representada pelos Estados de São Paulo e Rio de J aneiro tem maior destaque, posteriormente, a região sul e noroeste do Brasil. Isso vai ao encontro com o número de habitantes dessas regiões, bem como o número de instituições com Programa de PósGraduação em nível de mestrado e doutorado.

\section{A doença e a hospitalização no cotidiano da família de criança ou adolescente}

De acordo com a análise de conteúdo dos resumos emergiu a categoria a doença e a hospitalização no cotidiano de crianças e adolescentes. A família possui papel importante, no cuidado à criança, pois detém a responsabilidade com o bem-estar físico, emocional e social de seus membros, é no seio familiar que a criança tem seu referencial. ${ }^{15}$ Ainda, nesta perspectiva, pode-se afirmar que o cuidado centrado na família é importante, pois os profissionais são temporários na vida da criança, já a família é uma constante. ${ }^{16}$

Tratando-se de crianças com necessidades especiais de saúde, salienta-se que a doença crônica modifica a vida da criança/ adolescente, levando os mesmos a terem cuidados específicos com a saúde. Neste contexto, destaca-se a família, como principal cuidador, é no seio familiar que podem ser observadas as principais modificações após o diagnóstico. Autores afirmam que a família deve participar do processo de cuidado, isso requer do enfermeiro uma postura atenta às interações e aos impactos das vivências que ocorrem no ambiente hospitalar. ${ }^{11}$

A doença e a hospitalização alteram a dinâmica familiar, o estresse permeia as relações interpessoais, podendo até mesmo gerar uma crise entre os membros da família. Tratando-se de crianças hospitalizadas faz-se necessário ressaltar a importância das unidades pediátricas terem profissionais preparados para trabal har com pacientes pediátricos e com a família.

A hospitalização é estressante para a criança, envolvendo adaptações às mudanças que ocorrem no seu cotidiano. A criança depara-se com ambiente desconhecido, pessoas estranhas e procedimentos dolorosos, o que gera medo, ansiedade e insegurança. ${ }^{17-18}$

A doença gera uma ruptura na dinâmica familiar, pois a criança e a família ingressam em outro mundo, um mundo estranho, o mundo do hospital. A família passa a vivenciar outra dimensão do existir. Os seus projetos de futuro são ameaçados, passa a enfrentar a possibilidade de perda do filho e aflora uma gama de sentimentos como medo, culpa e, até mesmo, raiva. ${ }^{19}$ 


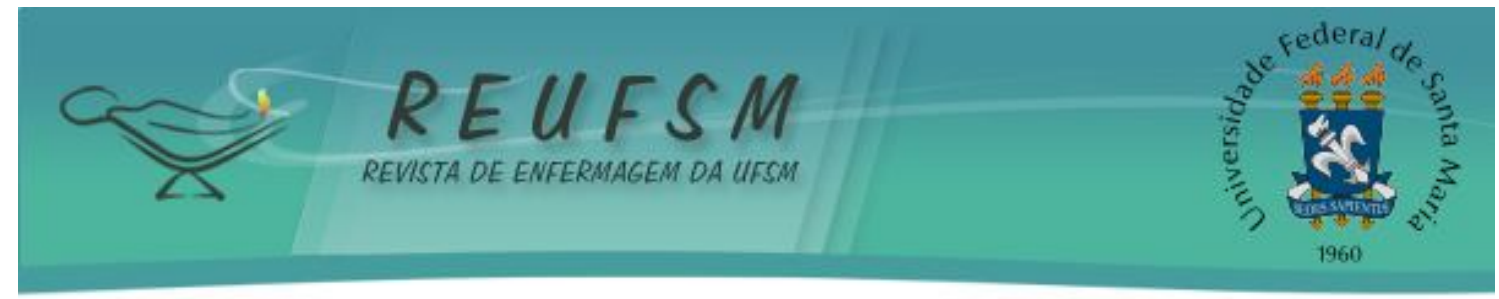

Diante das afirmações acima, percebe-se que é necessário atentar para os cuidados referentes à criança/adolescente hospitalizado, entretanto, deve-se incluir a família como cliente que necessita de atenção. O saber da família deve ser considerado, tendo em vista que a família é uma referência para a criança.

Neste sentido, a enfermagem deve ser preparada para trabalhar com famílias, considerando-se que no pós-alta hospitalar é o cuidado familiar que mantém a sobrevivência e a qualidade de vida da criança e/ ou adolescente.

\section{CONSIDERAÇÕES FINAIS}

Por meio da busca por produções que abordassem a criança com doença crônica e/ ou dependente de tecnologia, grupo em que se incluem as CRIANES, constatou-se que a tendência dos estudos está voltada para a família da criança doente, a doença e a hospitalização. Porém, identificaram-se lacunas no que tange ao preparo destas famílias para dar continuidade ao tratamento e aos cuidados no domicílio.

As crianças com doenças crônicas e/ ou incapacitantes possuem uma gama de cuidados especiais de saúde, neste sentido, faz-se necessário que a enfermagem esteja preparada para contemplar as demandas de cuidados à criança e/ ou adolescente que emergem durante a hospitalização a fim de que a criança tenha continuidade do tratamento no âmbito domiciliar.

Recomenda-se comprometimento e capacitação constante dos profissionais de saúde, principalmente, a equipe de enfermagem que necessitam atentar para essa clientela, assistindo as famílias que, repentinamente, se veem no papel de cuidador de uma CRIANES. Neste sentido, destaca-se o desenvolvimento do cuidado centrado na família que pode ser subsidiado por resultados de trabalhos investigativos desenvolvidos com esta clientela.

\section{REFERENCIAS}

1. Morel MF. L'enfant malade aux XVII et XIX siécles. In: Cook J, Domergues, J. L'enfant malades et lê mond medical: dialogue entre famille et soignants. Paris (FR): Syros; 1993. p.11-36.

2. Rocha SM. A criança na sociedade brasileira: educar, prevenir, cuidar. In: Anais do I Congresso Paulista de Enfermagem Pediátrica. São Paulo; 1995. p.3-7.

3. Ministério da Saúde (BR), Programa de Assistência Integral à Saúde da Criança (PAISC). Brasília (DF); 1984.

4. Ministério da Saúde (BR), Estatuto da Criança e do Adolescente. Lei 8.069 de 13 de Julho de 1990. Brasília (DF);2005.

5. Silva FD, Cabral IE. O cuidado de Enfermagem ao egresso da terapia intensiva: Reflexos na produção científica nacional de enfermagem pediátrica na década de 90 . Rev Eletrônica de Enferm (Goiânia) [periódico da internet]. 2001[citado 26 jan. 2010]; 3(2). Disponível em: http:// www. fen.ufg. br/ revista/ revista.html.

6. McPherson MG, Arango P, Fox H, Lauver C, Mc-Manus M, Newachek PW, et al. A new definition of children with special health care needs. Pediatrics. 1998;102(1):137-41.

7. Freire, P. Conscientização: teoria e prática da libertação: uma introdução ao pensamento de Paulo Freire. 3a ed. São Paulo: Centauro; 2005.

8. Góes FGB, La Cava AM. Práticas educativas em saúde do enfermeiro com a família da criança hospitalizada. Rev Eletrônica Enferm. 2009;11(4):942-51. 


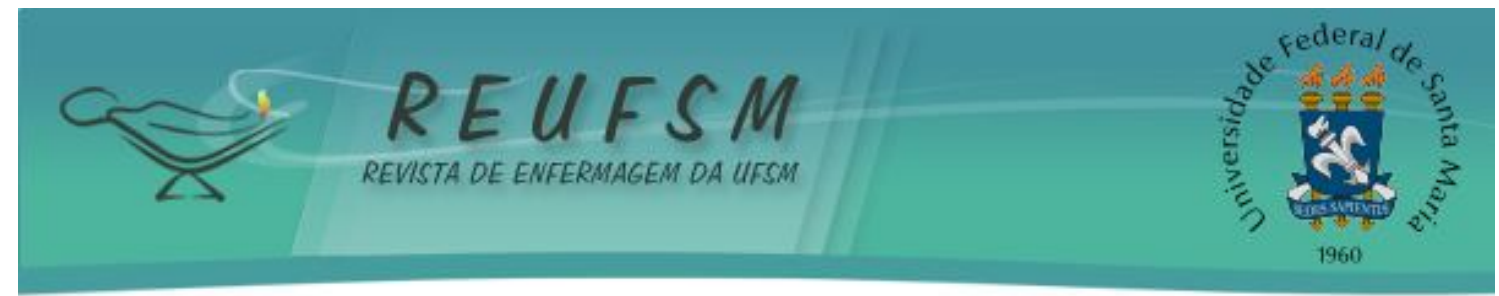

9. Queiroz MV, Jorge MS. Estratégias de educação em saúde e a qualidade do cuidar e ensinar em pediatria: a interação, o vínculo e a confiança no discurso dos profissionais. Interface (Botucatu). 2006; 10(19): 117-130.

10. Minayo MCS. O desafio do conhecimento: pesquisa qualitativa em saúde. 11ạ ed. São Paulo: HUCITEC; 2008.

11. Wernet $M$, Ângelo M. Mobilizando-se para a família: dando um novo sentido à família e ao cuidar. Rev Esc Enferm USP. 2003; 37(1): 19-25.

12. Turato ER. Métodos qualitativos e quantitativos na área da saúde: definições, diferenças e seus objetos de pesquisa. Rev Saúde Pública. 2005; 39(3):507-14.

13. Leopardi MT. Metodologia da pesquisa em saúde. Santa Maria: Pallotti; 2001.

14. Erdmann AL, Fernandes JV, Melo C, Carvalho BR, Menezes Q, Freitas R et al. A visibilidade da profissão de enfermeiro: reconhecendo conquistas e lacunas. Rev Bras Enferm. 2009; 62(4):637-43.

15. Comaru NRC, Monteiro ARM. O cuidado domiciliar à criança em quimioterapia na perspectiva do cuidador familiar. Rev Gaúcha Enferm. 2008; 29(3):423-30.

16. Wong DL. Enfermagem pediátrica: elementos essenciais à intervenção efetiva. 5a ed. Rio de J aneiro: Guanabara Koogan; 1999.

17. Moreira PL, Dupas G. Significado de saúde e de doença na percepção da criança. Rev Latino-Am Enfermagem. 2003; 11(6): 757-762.

18. Martins MR, Ribeiro CA, Borba RIH, Silva CV. Protocolo de preparo da criança préescolar para punção venosa, com utilização do brinquedo terapêutico. Rev Latino-Am Enfermagem. 2001; 9(2): 76-85.

19. Motta MGC. O ser doente no tríplice mundo da criança, família e hospital: uma descrição fenomenológica das mudanças existenciais [tese]. Florianópolis: Universidade Federal de Santa Catarina; 1997.

Data de recebimento: 26/01/2011

Data de aceite: 29/03/2011

Contato com autora responsável: Andressa da Silveira.

Endereço: Rua J oão Atílio Zampieri, 132/ 301, Bairro Camobi.

E-mail: andressadasilveira@gmail.com 